\title{
Apetite e sintomas de impacto nutricional de mulheres com câncer ginecológico
}

\author{
Appetite and nutritional impact symptoms of women with gynecological cancer
}

DOI: $10.37111 /$ braspenj.2020351006

Maria Claudia Bernardes Spexoto

Gesieli Aparecida da Silva²

Lígia Zampieri de Brito ${ }^{2}$

Mariana dos Santos Murra ${ }^{3}$

Ricardo Reis ${ }^{4}$

\section{Unitermos:}

Apetite. Neoplasias. Neoplasias dos genitais femininos. Estado nutricional.

\section{Keywords:}

Appetite. Neoplasms. Genital neoplasms, female. Nutritional status.

\section{Endereço para correspondência:}

Maria Claudia Bernardes Spexoto

Universidade Federal da Grande Dourados

Faculdade de Ciências da Saúde - Curso de Nutrição

Rodovia Dourados / Itahum, Km 12 - Unidade II -

Cidade Universitária - Caixa Postal: 364 - Dourados,

MS, Brasil - CEP: 79 804-970

E-mail: mariaspexoto@ufgd.edu.br

\section{Submissão}

4 de novembro de 2019

Aceito para publicação

21 de março de 2020

\section{RESUMO}

Introdução: Alterações no apetite são comuns e preocupantes nos pacientes com câncer e impactam de maneira significativa no estado nutricional. Este trabalho teve por objetivo verificar a relação entre apetite/sintomas e as características sociodemográficas, clínicas e do estado nutricional de mulheres diagnosticadas com câncer ginecológico e virgens de tratamento antineoplásico atendidas no Hospital de Câncer de Barretos. Método: Trata-se de um estudo transversal com delineamento amostral não probabilístico, realizado entre os meses de janeiro e setembro de 2017. Compuseram este estudo variáveis sociodemográficas, clínicas, estado nutricional e apetite/sintomas. Para a avaliação nutricional foi utilizado o índice de massa corporal (IMC) e a Avaliação Subjetiva Global Produzida pelo Próprio Paciente (ASG-PPP). Para avaliar o apetite e sintomas foi utilizado o instrumento Cancer Appetite and Symptom Questionnaire (CASQ). Para as associações de interesse foi utilizado o teste de qui-quadrado. Adotou-se nível de significância de 5\%. Resultados: Participaram 171 mulheres, com média de idade igual a 52,32 $\pm 14,95$ anos, sendo a maioria diagnosticada com neoplasia de colo de útero $(56,6 \%)$. Embora a predominância neste estudo seja de mulheres com o comprometimento moderado do apetite/sintomas, a mesma não apresentou relação com as variáveis sociodemográficas e clínicas, nem tampouco esteve associado ao estado nutricional avaliado pelo IMC. Entretanto, associação significativa foi encontrada entre o apetite/sintomas e o estado nutricional avaliado pela ASG-PPP $(p=0,033)$. Conclusão: $O$ comprometimento do apetite e presença de sintomas não apresentou relação com as variáveis sociodemográficas e clínicas estudadas nas mulheres diagnosticadas com câncer ginecológico virgens de tratamento. Associação significativa foi encontrada entre o CASQ e o estado nutricional avaliado pela ASG-PPP.

\section{ABSTRACT}

Introduction: Changes in appetite are common and worrying in cancer patients and significantly impact nutritional status. This study aimed to verify the relationship among appetite/symptoms and the sociodemographic, clinical and nutritional status of women diagnosed with gynecological cancer without antineoplastic treatment treated at Barretos Cancer Hospital. Methods: This is a cross-sectional study with a non-probabilistic sample design, conducted between January and September 2017. This study comprised sociodemographic, clinical, nutritional status and appetite/ symptoms. Nutritional assessment was performed using the body mass index (BMI) and the PatientGenerated Subjective Global Assessment (PG-SGA). To assess appetite and symptoms, the Cancer Appetite and Symptom Questionnaire (CASQ) instrument was used. For associations of interest, the chi-square test was used. A significance level of $5 \%$ was adopted. Results: 171 women with a mean age of $52.32 \pm 14.95$ years participated, most of them diagnosed with cervical cancer $(56.6 \%)$. Although the predominance in this study is women with moderate appetite/symptom impairment, it was not related to sociodemographic and clinical variables, nor was it associated with nutritional status assessed by BMI. However, a significant association was found between appetite/symptoms and nutritional status assessed by PG-SGA ( $p=0.033)$. Conclusion: Appetite impairment and presence of symptoms were not related to the sociodemographic and clinical variables studied in women diagnosed with treatment-naive gynecological cancer. Significant association was found between CASQ and nutritional status assessed by PG-SGA.

1. Professor Adjunto da Faculdade de Ciências da Saúde da Universidade Federal da Grande Dourados, Dourados, MS, Brasil.

2. Graduação em Nutrição, Centro Unificado de Educação Barretos Ltda., Barretos, SP, Brasil.

3. Nutricionista Clínica do Hospital de Amor, Barretos, SP, Brasil.

4. Médico Cirurgião, Departamento de Ginecologia Oncológica e Diretor da Área de Ensino do Instituto de Ensino e Pesquisa no Hospital de Amor, Barretos, SP, Brasil. 


\section{INTRODUÇ̃̃O}

O câncer ginecológico, especialmente o de colo do útero, também denominado cervical, é um dos mais incidentes entre as mulheres obesas, residentes em países em desenvolvimento e baixa condição socioeconômica. No Brasil, o câncer de colo do útero está representado com 16.370 casos novos, segundo as estimativas do Instituto Nacional do Câncer'.

As doenças malignas aumentam o nível de estresse metabólico e podem favorecer o processo de desnutrição, o que pode acometer o paciente ao diagnóstico, na descoberta da doença, e em qualquer fase do tratamento ${ }^{2,3}$.

$O$ reconhecimento precoce de pacientes em risco nutricional, bem como a determinação de fatores que impedem a ingestão adequada de alimento, como os sintomas de impacto nutricional (SIN), é fundamental para o manejo de uma nutrição adequada ${ }^{4}$. A ingestão insuficiente de alimentos é um problema comum em pacientes com câncer e está relacionado a vários fatores, como os SIN comumente relatados pelos pacientes, tamanho e localização do tumor, estadiamento clínico e toxicidades relacionadas aos tratamentos ${ }^{4}$.

Alterações do apetite são sintomas extremamente comuns em pacientes com câncer em risco de desnutrição e apresentam impacto significativo no estado nutricional e na qualidade de vida ${ }^{2,5-7}$.

Estas alterações podem se manifestar de diferentes maneiras, como a redução do desejo de consumir alimentos (anorexia), que é conhecida como causa primária para a redução da ingestão de alimentos ${ }^{3,5}$, a saciedade precoce ou a combinação de ambas manifestações, sendo este último o pior cenário relatado8. Quando diagnosticadas precocemente e iniciado tratamento específico para alívio dos sintomas, os seus efeitos sobre o estado nutricional podem ser minimizados.

As causas da falta de apetite são as mais diversas, entre elas a inflamação sistêmica gerada pelo próprio tumor ${ }^{2}$, os tratamentos antineoplásicos ${ }^{9,10}$, alterações anatômicas após procedimentos cirúrgicos, deficiências de micronutrientes, e não menos importante, a ansiedade e depressão, até mesmo ao diagnóstico, podem corroborar na supressão do apetite $^{11,12}$

É bem conhecido que as alterações do apetite impactam no estado nutricional de pacientes com câncer em tratamento, mas pouco se investiga sobre a sua existência e sua relação com o estado nutricional de pacientes recém diagnosticados, nem tampouco com mulheres diagnosticadas com tumores ginecológicos e ainda virgens de tratamento antineoplásico.

Investigar a falta de apetite, a presença de sintomas e o estado nutricional é fundamental em todas as etapas do tratamento do câncer. Deste modo, é de extrema importância o uso de instrumentos de avaliação, que além de válidos e confiáveis, sejam de fácil utilização como, por exemplo, - Cancer Appetite Symptom Questionnaire (CASQ) ${ }^{13,14}$, para avaliação do comprometimento do apetite/sintomas, e a Avaliação Subjetiva Global (ASG) ${ }^{15}$, para diagnóstico nutricional.
Desta forma, o presente estudo tem por objetivo verificar a relação entre o apetite/sintomas e as características sociodemográficas, clínicas e do estado nutricional de mulheres diagnosticadas com câncer ginecológico.

\section{MÉTODO}

\section{Desenho de Estudo e Delineamento Amostral}

Trata-se de estudo transversal com delineamento amostral não probabilístico. Foram convidadas a participar e incluídas no estudo todas as mulheres diagnosticadas com câncer ginecológico entre os meses de janeiro e setembro de 2017, no Hospital de Amor (Hospital de Câncer de Barretos).

\section{Critérios de Elegibilidade}

Foram incluídas mulheres com idade igual ou maior que 20 anos, com diagnóstico de câncer ginecológico confirmado, sem quaisquer tratamentos prévios, que concordaram e assinaram o Termo de Consentimento Livre e Esclarecido (TCLE).

Foram excluídas as mulheres com déficit mental ou cognitivo que impossibilitassem o preenchimento dos questionários do estudo e/ou a avaliação antropométrica.

\section{Variáveis de Estudo}

Compuseram este estudo informações sociodemográficas, clínica, avaliação do estado nutricional e apetite/sintomas.

As informações sociodemográficas, variáveis do estado nutricional e apetite/sintomas foram levantadas por meio de entrevista pessoal. As variáveis clínicas, referentes às doenças, foram obtidas por meio de consulta ao prontuário das pacientes.

As variáveis sociodemográficas foram idade (anos completos), escolaridade (analfabeta, sabe ler e escrever, ensino fundamental incompleto, ensino fundamental completo, ensino médio incompleto, ensino médio completo, superior incompleto, superior completo e pós-graduação), estado civil (solteira, casada, desquitada/divorciada ou viúva), presença de atividade laboral (presente/ausente), nível econômico classificado segundo o Critério de Classificação Econômica Brasil'16, autodeclaração de raça, tabagismo e etilismo ('não', 'sim' ou ex-tabagista/ex-etilista).

Como variáveis clínicas foram investigados apenas o sítio do tumor ginecológico e o estadiamento clínico (I, II, III e IV). No presente estudo, os estadiamentos I e II foram denominados "não avançados" e os estadiamentos III e IV, "avançados".

\section{Estado Nutricional}

Para a avaliação do estado nutricional foram utilizadas as medidas de peso $(\mathrm{kg})$ e estatura $(\mathrm{m})$ para cômputo do índice de massa corporal (IMC) e ASG proposta por Ottery ${ }^{17}$. Utilizou-se balança digital, com capacidade $200 \mathrm{~kg}$, com estadiômetro acoplado ao equipamento da marca Toledo Prix ${ }^{\circledR}$. Foram adotados os critérios e pontos de corte propostos pela Organização Mundial da Saúde (OMS) ${ }^{18}$. 
Adotou-se a Avaliação Subjetiva Global Produzida pelo Próprio Paciente (ASG-PPP) adaptada culturalmente para o português por Campos e Prado ${ }^{19}$.

A primeira parte do instrumento foi preenchida pelo próprio paciente e/ou acompanhante e a segunda parte por um avaliador treinado em estudo piloto. Os pacientes foram classificados de acordo com a proposta de Ottery ${ }^{17}$ em "Bem nutrido (A)", "Moderadamente desnutrido (B)" e "Gravemente desnutrido (C)".

Neste estudo, as pacientes que apresentaram algum grau de desnutrição foram agrupadas numa mesma categoria (B e C).

\section{Apetite e Sintomas}

Para a avaliação do apetite e sintomas foi utilizado o instrumento $C A S Q$, proposto por Halliday et al. ${ }^{13}$, cuja tradução e adaptação transcultural para a língua portuguesa foi realizada por Spexoto et al. ${ }^{14}$.

Trata-se de um instrumento unifatorial, composto por 10 itens com resposta disposta em escala do tipo de Likert de cinco pontos (0-4), exceto o item 12 (referente à "dor"), que possui seis pontos de resposta. Ainda para o item 12, as respostas "sem dor" e "muito leve" foram compiladas numa mesma categoria denominada "sem dor e/ou muito leve". Cabe ressaltar que quatro itens do instrumento possuem escala de resposta invertida.

O cômputo do escore global de apetite e sintomas foi efetuado utilizando um algoritmo proposto pelos mesmos autores, conforme a Equação 1.

$$
\begin{gathered}
\text { Escore global apetite/sintomas } \\
=0,171 \text { it } 1+0,125 \text { it } 2+0,026 \text { it } 3+0,138 \text { it } 4+0,153 \text { it } 7+0,084 \text { it8 } \\
+0,117 \text { it } 9+0,047 \text { it } 10+0,101 \text { it } 11+0,053 \text { it } 12
\end{gathered}
$$

Equação 1 - Cômputo do escore global de apetite/ sintomas. It = item do instrumento CASQ.

Após o cômputo do escore global, as pacientes foram categorizadas em "Baixo comprometimento do apetite" (escore $\leq 1$ ), "Moderado comprometimento do apetite" (escore 1-3) e "Grave comprometimento do apetite" (escore $>3$ ).

\section{Aspectos Éticos}

Este estudo obedeceu às normas e diretrizes das Boas Práticas Clínicas, de acordo com a Resolução 466/2012 e foi aprovado no Comitê de Ética em Pesquisa do Hospital de Câncer de Barretos, sob o número do CAAE: 63515317.1 .0000 .5437$.

\section{Análise dos Dados}

Foi realizada análise descritiva dos dados por meio das medidas de tendência central e dispersão para as variáveis quantitativas. Para as variáveis qualitativas, utilizou-se tabelas contendo valores absolutos e relativos das mesmas.

As medidas de resumo e de forma da distribuição das respostas dadas ao CASQ foram utilizadas para estimar a sensibilidade psicométrica dos itens, onde itens com coeficiente de assimetria (Sk) superiores a 3 e de curtose (Ku) superiores a 7 foram considerados como apresentando problemas de sensibilidade.

Para verificar a relação entre o apetite/sintomas e as características sociodemográficas, clínicas e do estado nutricional foi utilizado o teste de qui-quadrado.

As análises foram realizadas com auxílio do programa SPSS versão 22 e adotou-se o nível de significância de 5\%.

\section{RESULTADOS}

Participaram do estudo 171 mulheres atendidas no Hospital de Câncer de Barretos, com média de idade igual a 52,32 $\pm 14,95$ anos, sendo a maioria diagnosticada com neoplasia de colo do útero $(56,6 \% ; n=94)$, com ausência de atividade laboral $(63,2 \%)$, casada $(52,6 \%)$, não tabagista $(71,9 \%)$, não etilista $(67,3 \%)$ e com excesso de peso ao IMC $(69,6 \%)$. Houve predomínio da raça branca $(49,1 \%)$ e escolaridade ensino fundamental incompleto $(34,5 \%)$.

Na amostra estudada, o escore global médio do apetite/ sintomas foi igual a 1,35 $\pm 0,63$ (mínimo $=0,35$, máximo $=3,39$ ). Portanto, as mulheres encontram-se com moderado comprometimento do apetite e presença de sintomas.

A distribuição de frequências de respostas dadas ao instrumento CASQ está apresentada na Tabela 1.

Nota-se que, quando questionadas sobre 0 apetite (it1 "Meu apetite é..."), uma quantidade expressiva de mulheres relatou apresentar algum grau de comprometimento do apetite, expressos em médio, pouco e muito pouco, representando $22,8 \%, 15,8 \%$ e 10,5\%, respectivamente.

A maioria das mulheres não possui saciedade precoce, sendo observado no it2, onde 73,4\% sentem-se satisfeitas após comer uma refeição completa.

A prevalência foi de mulheres que gostam da comida que comem a maioria das vezes $(70,2 \%)$, que o sabor está tão bom quanto antes de ficarem doentes $(77,6 \%)$ e que não apresentam nenhuma alteração no paladar $(73,1 \%)$.

Com relação à fadiga, representada pelo it 1 1, grande parte das pacientes $(40,9 \%)$ referiu grau moderado de energia.

Ainda na Tabela 1, destaca-se 0 it 12, referente à dor, onde $68,4 \%$ das pacientes relataram algum grau de dor (muito leve a intensa).

A Tabela 2 apresenta as medidas de resumo dos itens componentes do $C A S Q$, segundo as respostas dadas pelas participantes.

Nota-se que nenhum item apresentou violação importante em relação à normalidade, o que aponta adequada sensibilidade dos itens. 
Tabela 1 - Distribuição de frequências de respostas dadas ao instrumento Cancer Appetite and Symptom Questionnaire (CASQ).

\begin{tabular}{|c|c|c|c|c|}
\hline Item $^{*}$ & Resposta & & $\mathrm{n}$ & $\%$ \\
\hline \multirow[t]{6}{*}{ it1 Meu apetite é... } & Muito pouco & & 18 & 10,5 \\
\hline & Pouco & & 27 & 15,8 \\
\hline & Médio & & 39 & 22,8 \\
\hline & Bom & & 56 & 32,7 \\
\hline & Muito bom & & 31 & 18,1 \\
\hline & & Total & 171 & 100,0 \\
\hline \multirow[t]{6}{*}{ it2 Quando eu como eu me sinto cheio... } & Sem ter comido nada & & 4 & 2,4 \\
\hline & Depois de comer apenas um pouco & & 12 & 7,1 \\
\hline & Depois de comer cerca de um terço duma refeição & & 10 & 5,9 \\
\hline & Depois de comer mais da metade duma refeição & & 19 & 11,2 \\
\hline & Depois de comer uma refeição completa & & 124 & 73,4 \\
\hline & & Total & 169 & 100,0 \\
\hline \multirow[t]{6}{*}{ it3 $\quad$ Antes de comer eu sinto fome... } & Raramente & & 44 & 25,7 \\
\hline & Ocasionalmente & & 63 & 36,8 \\
\hline & Boa parte do tempo & & 26 & 15,2 \\
\hline & A maior parte do tempo & & 24 & 14,0 \\
\hline & O tempo todo & & 14 & 8,2 \\
\hline & & Total & 171 & 100,0 \\
\hline \multirow[t]{6}{*}{ it4 Eu gosto da comida que eu como... } & Nunca & & 2 & 1,2 \\
\hline & Raramente & & 8 & 4,7 \\
\hline & Algumas vezes & & 21 & 12,3 \\
\hline & Frequentemente & & 20 & 11,7 \\
\hline & A maioria das vezes & & 120 & 70,2 \\
\hline & & Total & 171 & 100,0 \\
\hline \multicolumn{5}{|l|}{ it7 Comparativamente a antes de estar } \\
\hline \multirow[t]{6}{*}{ doente, o sabor da comida é... } & Muito pior & & 9 & 5,3 \\
\hline & Pior & & 25 & 14,7 \\
\hline & Tão bom quanto antes & & 132 & 77,6 \\
\hline & Melhor & & 3 & 1,8 \\
\hline & Muito melhor & & 1 & 0,6 \\
\hline & & Total & 170 & 100,0 \\
\hline \multirow[t]{6}{*}{ it8 Atualmente eu tenho... } & Nenhum paladar & & 5 & 2,9 \\
\hline & Alteração importante no paladar & & 9 & 5,3 \\
\hline & Alteração moderada no paladar & & 12 & 7,0 \\
\hline & Alteração leve no paladar & & 20 & 11,7 \\
\hline & Nenhuma alteração no paladar & & 125 & 73,1 \\
\hline & & Total & 171 & 100,0 \\
\hline \multicolumn{5}{|l|}{ it9 Eu me sinto doente ou enjoado antes de } \\
\hline \multirow[t]{6}{*}{ comer ou quando como... } & Na maioria das vezes & & 13 & 7,6 \\
\hline & Frequentemente & & 8 & 4,7 \\
\hline & Algumas vezes & & 20 & 11,7 \\
\hline & Raramente & & 12 & 7,0 \\
\hline & Nunca & & 118 & 69,0 \\
\hline & & Total & 171 & 100,0 \\
\hline \multirow[t]{6}{*}{ It10 A maior parte do tempo, o meu humor é... } & Muito triste & & 16 & 9,4 \\
\hline & Triste & & 35 & 20,5 \\
\hline & Nem triste nem feliz & & 51 & 29,8 \\
\hline & Feliz & & 60 & 35,1 \\
\hline & Muito feliz & & 9 & 5,3 \\
\hline & & Total & 171 & 100,0 \\
\hline \multirow[t]{6}{*}{ it11 Na maioria das vezes, o meu nível de energia é... } & Muito baixo & & 17 & 9,9 \\
\hline & Baixo & & 44 & 25,7 \\
\hline & Moderado & & 70 & 40,9 \\
\hline & Alto & & 28 & 16,4 \\
\hline & Muito alto & & 12 & 7,0 \\
\hline & & Total & 171 & 100,0 \\
\hline \multirow[t]{7}{*}{ it12 A maior parte do tempo, minha dor é... } & Sem dor & & 54 & 31,6 \\
\hline & Muito leve & & 9 & 5,3 \\
\hline & Leve & & 19 & 11,1 \\
\hline & Moderada & & 49 & 28,7 \\
\hline & Intensa & & 23 & 13,5 \\
\hline & Muito intensa & & 17 & 9,9 \\
\hline & & Total & 171 & 100,0 \\
\hline
\end{tabular}


Tabela 2 - Medidas de resumo dos itens componentes do CASQ, segundo as respostas dadas pelas participantes.

\begin{tabular}{lccccccc}
\hline CASQ & $\mathbf{n}$ & Mínimo & Máximo & Média & Desvio padrão & Assimetria & Curtose \\
\hline it1 & 171 & 0 & 4 & 1,68 & 1,24 & 0,39 & $-0,83$ \\
it2 & 169 & 0 & 4 & 0,54 & 1,04 & 1,92 & 2,58 \\
it3 & 171 & 0 & 4 & 2,58 & 1,24 & $-0,65$ & $-0,60$ \\
it4 & 171 & 0 & 4 & 0,55 & 0,96 & 1,66 & 1,82 \\
it7 & 170 & 0 & 3 & 2,22 & 0,59 & 1,27 & 3,39 \\
it8 & 171 & 0 & 4 & 0,53 & 1,03 & 1,98 & 3,01 \\
it9 & 171 & 0 & 4 & 0,75 & 1,27 & 1,52 & 0,97 \\
it10 & 171 & 0 & 4 & 1,94 & 1,07 & 0,33 & $-0,68$ \\
it11 & 171 & 0 & 4 & 2,15 & 1,04 & $-0,15$ & $-0,31$ \\
it12 & 171 & 0 & 4 & 1,49 & 1,36 & 0,35 & $-1,10$ \\
\hline
\end{tabular}

It = item do instrumento CASQ.

A Tabela 3 apresenta a relação entre o apetite/sintomas, avaliado pelo instrumento $C A S Q$, e as características sociodemográficas, clínicas e o estado nutricional pelas categorias do IMC e ASG-PPP.

A maioria das mulheres encontrava-se bem nutrida (A) $(84,1 \%)$. A desnutrição estava presente em $15,9 \%$ das pacientes.
As variáveis sociodemográficas e clínicas não apresentaram relação com o comprometimento do apetite/sintomas, nem tampouco este esteve associado com o IMC.

$\bigcirc$ apetite e sintomas apresentaram associação significativa com o estado nutricional avaliado pela ferramenta $\operatorname{ASG-PPP}\left(\chi^{2}=10,469 ; p=0,033\right)$.

Tabela 3 - Relação entre o apetite/sintomas e as características sociodemográficas, clínica e estado nutricional.

\begin{tabular}{|c|c|c|c|c|c|}
\hline \multirow[b]{2}{*}{ Variáveis } & \multicolumn{5}{|c|}{ Apetite e Sintomas } \\
\hline & Baixo & Moderado & Grave & $\mathrm{x}^{2}$ & $p$ \\
\hline \multicolumn{6}{|l|}{ Sociodemográficas } \\
\hline Analfabeto & 4 & 6 & 2 & 23,418 & 0,103 \\
\hline Sabe ler e escrever & 2 & 8 & - & & \\
\hline Ensino fundamental incompleto & 22 & 36 & 1 & & \\
\hline Ensino médio incompleto & 1 & 4 & - & & \\
\hline Ensino médio completo & 16 & 17 & - & & \\
\hline Superior incompleto & 1 & 9 & - & & \\
\hline Superior completo & 7 & 4 & - & & \\
\hline Pós-graduação & 1 & 3 & - & & \\
\hline \multicolumn{6}{|l|}{ Estado civil } \\
\hline Solteira & 14 & 20 & 1 & 3,932 & 0,686 \\
\hline Casada & 34 & 53 & 2 & & \\
\hline Viúva & 11 & 15 & - & & \\
\hline Desquitada/divorciada & 4 & 15 & 1 & & \\
\hline \multicolumn{6}{|l|}{ Raça } \\
\hline Branca & 32 & 50 & 1 & 1,836 & 0,766 \\
\hline Negra & 5 & 9 & 1 & & \\
\hline Parda & 26 & 44 & 2 & & \\
\hline \multicolumn{6}{|l|}{ Tabagista } \\
\hline
\end{tabular}




\begin{tabular}{|c|c|c|c|c|c|}
\hline CASQ & \multicolumn{5}{|c|}{ Apetite e Sintomas } \\
\hline Variáveis & Baixo & Moderado & Grave & $\mathrm{x}^{2}$ & $p$ \\
\hline \multicolumn{6}{|l|}{ Etilista } \\
\hline Não & 41 & 69 & 4 & 7,119 & 0,130 \\
\hline $\operatorname{Sim}$ & 9 & 24 & - & & \\
\hline Ex-etilista & 13 & 10 & - & & \\
\hline \multicolumn{6}{|l|}{ Nível econômico* } \\
\hline A & 2 & 1 & - & 6,375 & 0,382 \\
\hline B & 14 & 18 & 1 & & \\
\hline C & 34 & 49 & 3 & & \\
\hline DeE & 12 & 34 & - & & \\
\hline \multicolumn{6}{|l|}{ Clínica } \\
\hline \multicolumn{6}{|l|}{ Sítio do tumor } \\
\hline Colo do útero & 34 & 58 & 2 & 7,767 & 0,457 \\
\hline Endométrio & 18 & 19 & 2 & & \\
\hline Ovário & 8 & 19 & - & & \\
\hline Vagina & - & 3 & - & & \\
\hline Vulva & - & 2 & - & & \\
\hline \multicolumn{6}{|l|}{ Estadiamento clínico } \\
\hline Não avançado (I e ll) & 31 & 52 & 1 & 1,053 & 0,591 \\
\hline Avançado (III e IV) & 19 & 38 & 2 & & \\
\hline \multicolumn{6}{|l|}{ Estado nutricional } \\
\hline \multicolumn{6}{|l|}{ IMC } \\
\hline Baixo peso & 3 & 2 & 1 & 8,144 & 0,228 \\
\hline Eutrofia & 16 & 29 & 1 & & \\
\hline Pré-obesidade & 23 & 35 & - & & \\
\hline Obesidade & 21 & 37 & 2 & & \\
\hline \multicolumn{6}{|l|}{ Avaliação Subjetiva Global } \\
\hline Bem nutrido $(A)$ & 58 & 83 & 2 & 10,469 & 0,033 \\
\hline Desnutrido (B e C) & 5 & 20 & 2 & & \\
\hline
\end{tabular}

\section{DISCUSSÃO}

Este estudo contribuiu em verificar que o comprometimento do apetite e SIN podem estar presentes em mulheres diagnosticadas com câncer ginecológico, com predominância em sítio colo uterino, antes mesmo de iniciarem o tratamento antineoplásico. Isso vem para chamar a atenção e reforçar aos profissionais de saúde da área de oncologia sobre a importância de rastrear o risco e iniciar o cuidado nutricional precocemente, e que estes sejam realizados de rotina no ambulatório da ginecologia oncológica, e não somente por demanda dos médicos e/ ou enfermeiros.

Além disso, neste estudo encontramos que o apetite/ sintomas apresentou associação significativa com o estado nutricional diagnosticado pela ASG-PPP. Em estudo conduzido por Nho et al. ${ }^{12}$, o apetite foi um dos fatores significativos preditivos para desnutrição de pacientes com câncer ginecológico.
A desnutrição esteve presente em apenas 15,9\% das participantes. Mas é importante ressaltar que, as mesmas seriam submetidas aos tratamentos antineoplásicos e, sejam eles quais forem, a desnutrição já as colocaria em um cenário desfavorável com prejuízos metabólicos, nutricionais e poderia comprometer o protocolo terapêutico. Em um estudo de coorte conduzido por Laky et al. ${ }^{20}$, que envolveu 157 pacientes com diagnóstico suspeito ou confirmado de câncer ginecológico, foram submetidas à ASG-PPP antes de iniciarem o tratamento, e observaram que $24,8 \%$ dos casos já apresentavam algum grau de desnutrição (B e C), sendo esta condição um dos fatores pré-cirúrgicos que apresentou associação significativa com o tempo prolongado de internação hospitalar.

O comprometimento do apetite e a presença de SIN não apresentaram relação com as variáveis sociodemográficas, clínicas e IMC. De fato, esperávamos encontrar neste estudo relação entre o apetite/sintomas e o estadiamento clínico, pois 
é conhecido que os pacientes que apresentam câncer avançado possuem mais sintomas que impactam no estado nutricional $^{3,21}$, mesmo que em quantidade e gravidade distintas. No nosso estudo, grande parte da amostra foi diagnosticada de forma tardia nos estadiamentos III e IV. Talvez esta relação não tenha sido encontrada pois os sintomas predominantes do câncer de colo do útero, o qual foi diagnóstico da maioria das participantes deste estudo, geralmente são sangramento vaginal intermitente ou após relação sexual, secreção vaginal anormal e dor abdominal inferior associada a queixas urinárias ou intestinais ${ }^{22}$, os quais não foram investigados neste estudo, exceto a presença de dor.

Embora a predominância neste estudo seja de pacientes classificadas com excesso de peso ao IMC e bem nutridas, estas já apresentavam comprometimento do apetite/presença de sintomas moderado, avaliado por meio do escore global do CASQ. Os SIN geralmente são mais incidentes em pacientes desnutridos e/ou com outras complicações nutricionais ${ }^{23}$, mas os achados deste estudo reforçam que os SIN podem surgir antes mesmo do início do tratamento $\mathrm{e}$, inclusive, em pacientes com excesso de peso.

Tal fato pode ser justificado mediante ao processo inflamatório sistêmico, sendo o tumor por si só o principal responsável por esta condição. Este, por sua vez, pode contribuir às mudanças importantes no comportamento do apetite em virtude do aumento na produção de citocinas próinflamatórias (a exemplo, as interleucinas 1-IL-1 e 6-IL-6, e o fator de necrose tumoral alfa - TNF- $\alpha$ ), e esta cascata de citocinas pode afetar o controle neuroendócrino do apetite, levando à anorexia ${ }^{3,5}$.

Neste estudo, a maioria das mulheres entrevistadas gosta da comida que come na maioria das vezes, não apresenta queixas relacionadas ao sabor dos alimentos, bem como não apresenta nenhuma alteração no paladar. Isso pode ter ocorrido em virtude das participantes serem recém diagnosticadas, virgens de quaisquer tratamentos do câncer e não experimentarem os efeitos colaterais relacionados ao tratamento. Mesmo assim, reforçamos que as pacientes apresentaram moderado comprometimento do apetite e isso pode ser um alerta à equipe multidisciplinar para $\mathrm{O}$ rastreamento precoce da falta de apetite e SIN, se possível, previamente ao tratamento, de forma a minimizar a perda de peso e impactos negativos sobre o estado nutricional.

A maioria das pacientes relatou algum grau de dor (muito leve a intensa). Sabe-se que a dor é um $\mathrm{SIN}^{4}$, a qual pode contribuir de forma significativa no estado nutricional.

Fica evidente neste estudo que uma abordagem nutricional baseada apenas no IMC está fracassada quando utilizado de forma isolada. Frente à epidemia global da obesidade e à nova compreensão das alterações metabólicas que podem ocorrer antes de quaisquer alterações no peso corporal, faz-se necessária a incorporação na prática clínica da avaliação do apetite/sintomas e a utilização da ASG ou ASG-PPP para diagnóstico nutricional de pacientes com câncer, conforme recomendado por entidades e especialistas ${ }^{24,25}$.

Hébuterne et al. ${ }^{26}$ conduziram um estudo prospectivo de prevalência de 1 dia, realizado em 154 enfermarias públicas ou privadas em 24 cidades francesas e, embora tenham utilizado o IMC para o diagnóstico nutricional, os autores apresentaram uma amostra robusta. Foram avaliados 1903 pacientes, sendo 39\% dos pacientes desnutridos na amostra global. Nas pacientes com câncer de ovário/útero ( $n=87)$, com média de idade semelhante ao nosso estudo, $41 \%$ encontravam-se desnutridas. Os autores recomendam rastreamento sistemático e tratamento da desnutrição. Cabe salientar que, os autores avaliaram também a perda de peso não intencional dos pacientes, uma vez que consideramos esta informação fundamental, tanto no rastreamento do risco quanto para o diagnóstico nutricional.

Para tanto, recomenda-se a utilização do CASQ como componente da avaliação do cuidado nutricional com intuito de proporcionarmos suporte nutricional antes mesmo da perda de peso acontecer, e estabelecermos intervenções individualizadas para uma ingestão alimentar segura e atenuação do estresse metabólico e processo inflamatório.

Este estudo apresenta algumas limitações que devem ser apontadas. A primeira refere-se ao tamanho amostral, que embora seja expressivo, não representa a totalidade de mulheres atendidas pelo departamento de ginecologia oncológica no ano de 2017, o que limita a extrapolação dos resultados. Entretanto, os nossos achados reforçam a importância da identificação precoce do risco nutricional destas pacientes, antes mesmo de iniciarem o tratamento antineoplásico. A segunda tange ao desenho do estudo transversal, o qual não permite estabelecer relação direta de causa e efeito. Apesar destas limitações, este estudo se preocupou em investigar o estado nutricional, o apetite e os sintomas de impacto nutricional em mulheres recém diagnosticadas com tumores ginecológicos, antes mesmo de iniciarem quaisquer tratamentos propostos, o que possivelmente venha a reforçar aos pesquisadores e profissionais da área a necessidade de rastreamento destas informações o quanto antes.

\section{CONCLUSÃO}

O comprometimento do apetite/sintomas avaliado pelo instrumento CASQ não apresentou relação com as variáveis sociodemográficas e clínicas estudadas nas mulheres diagnosticadas com câncer ginecológico. Associação significativa foi encontrada entre as categorias do CASQ e o estado nutricional avaliado pela ASG-PPP. 


\section{REFERÊNCIAS}

1. INCA. Estimativa 2018: incidência de câncer no Brasil. Rio de Janeiro: Instituto Nacional de Câncer José Alencar Gomes da Silva; 2017.

2. Fearon K, Strasser F, Anker SD, Bosaeus I, Bruera E, Fainsinger $\mathrm{RL}$, et al. Definition and classification of cancer cachexia: an international consensus. Lancet Oncol. 2011;12(5):489-95.

3. Arends J, Baracos V, Bertz H, Bozzetti F, Calder PC, Deutz NEP, et al. ESPEN expert group recommendations for action against cancer-related malnutrition. Clin Nutr. 2017;36(5):1187-96.

4. Omlin A, Blum D, Wierecky J, Haile SR, Ottery FD, Strasser F. Nutrition impact symptoms in advanced cancer patients: frequency and specific interventions, a case-control study. J Cachexia Sarcopenia Muscle. 2013;4(1):55-61.

5. Fearon K, Arends J, Baracos V. Understanding the mechanisms and treatment options in cancer cachexia. Nat Rev Clin Oncol. 2013;10(2):90-9.

6. Campos JA, Silva WR, Spexoto MC, Serrano SV, Marôco J. Clinical, dietary and demographic characteristics interfering on quality of life of cancer patients. Einstein (Sao Paulo). 2018;16(4):1-9.

7. Quinten C, Coens C, Mauer M, Comte S, Sprangers MA, Cleeland $\mathrm{C}$, et al. Baseline quality of life as a prognostic indicator of survival: a meta-analysis of individual patient data from EORTC clinical trials. Lancet Oncol. 2009;10(9):865-71.

8. Barajas Galindo DE, Vidal-Casariego A, Calleja-Fernández A, Hernández-Moreno A, Pintor de la Maza B, Pedraza-Lorenzo M, et al. Appetite disorders in cancer patients: impact on nutritional status and quality of life. Appetite. 2017;114:23-7.

9. Ogama N, Suzuki S, Umeshita K, Kobayashi T, Kaneko S, Kato $\mathrm{S}$, et al. Appetite and adverse effects associated with radiation therapy in patients with head and neck cancer. Eur J Oncol Nurs. 2010;14(1):3-10.

10. Ogama N, Ogama N. Development of an oral assessment tool to evaluate appetite in patients with head and neck cancer receiving radiotherapy. Eur J Oncol Nurs. 2013;17(4):474-81.

11. Tisdale MJ. Cancer cachexia. Curr Opin Gastroenterol. 2010;26(2):146-51.

12. Nho JH, Kim SR, Kwon YS. Depression and appetite: predictors of malnutrition in gynecologic cancer. Support Care Cancer. 2014;22(11):3081-8.

13. Halliday V, Porock D, Arthur A, Manderson C, Wilcock A. Development and testing of a cancer appetite and symptom questionnaire. J Hum Nutr Diet. 2012;25(3):217-24.

14. Spexoto MC, Serrano SV, Halliday V, Maroco J, Campos JA. Cancer Appetite and Symptom Questionnaire (CASQ) for Brazilian patients: cross-cultural adaptation and validation study. PLoS One. 2016;11(6):e0156288.
15. Detsky AS, McLaughlin JR, Baker JP, Johnston N, Whittaker $\mathrm{S}$, Mendelson RA, et al. What is subjective global assessment of nutritional status? JPEN J Parenter Enteral Nutr. 1987;11(1):8-13.

16. Associação Brasileira de Empresas de Pesquisa. Critério Brasil 2015 e atualização da distribuição de classes para 2016. Critério de Classificação Econômica Brasil. [cited 2019 Oct 12]. Available from: http://www.abep.org/criterio-brasil

17. Ottery FD. Definition of standardized nutritional assessment and interventional pathways in oncology. Nutrition. 1996;12(1 Suppl):S15-9.

18. World Health Organization. Obesity: preventing and managing the global epidemic [Internet]. Vol. 894, World Health Organization technical report series. Geneva: World Health Organization; 2000. [cited 2019 Oct 12]. Available from: http://books.google. com/books?hl=en\&lr=\&id=AvnqOsqv9doC\&oi=fnd\&pg=PA $1 \& \mathrm{dq}=$ Obesity: + Preventing + and + managing + the + global + epi demic\&ots=6UF0cjXY6M\&sig $=\mathrm{iA} 1 \mathrm{~h} 8 \mathrm{eoKNhRTKDfXqIVZ}$ aivyyk4\%5Cnhttp://www.ncbi.nlm.nih.gov/pubmed/11234459

19. Campos JADB, Prado CD. Cross-cultural adaptation of the Portuguese version of the Patient-Generated Subjective Global Assessment. Nutr Hosp. 2012;27(2):583-9.

20. Laky B, Janda M, Kondalsamy-Chennakesavan S, Cleghorn G, Obermair A. Pretreatment malnutrition and quality of life - association with prolonged length of hospital stay among patients with gynecological cancer: a cohort study. BMC Cancer. 2010;10:232.

21. Cederholm T, Jensen GL. To create a consensus on malnutrition diagnostic criteria: a report from the Global Leadership Initiative on Malnutrition (GLIM) meeting at the ESPEN Congress 2016. Clin Nutr. 2017;36(1):7-10.

22. Mwaka AD, Okello ES, Wabinga H, Walter FM. Symptomatic presentation with cervical cancer in Uganda: a qualitative study assessing the pathways to diagnosis in a low-income country. BMC Womens Health. 2015;15:15.

23. Brasil. Consenso nacional de nutrição oncológica. $2^{\mathrm{a}}$ ed. rev. Rio de Janeiro: Ministerio da Saúde, Instituto Nacional de Câncer; 2015.

24. Horie LM, Barrére APN, Castro MG, Liviera AMB, Carvalho AMB, Pereira A, et al. Diretriz BRASPEN de terapia nutricional no paciente com câncer. BRASPEN J. 2019;34(Supl 1):2-32.

25. Jager-Wittenaar H, Ottery FD. Assessing nutritional status in cancer: role of the Patient-Generated Subjective Global Assessment. Curr Opin Clin Nutr Metab Care. 2017;20(5):322-9.

26. Hébuterne $X$, Lemarié E, Michallet M, De Montreuil CB, Schneider SM, Goldwasser F. Prevalence of malnutrition and current use of nutrition support in patients with cancer. JPEN J Parenter Enteral Nutr. 2014;38(2):196-204.

Local de realização do estudo: Hospital de Amor (Hospital de Câncer de Barretos), Barretos, SP, Brasil.

Conflito de interesse: Os autores declaram não haver. 